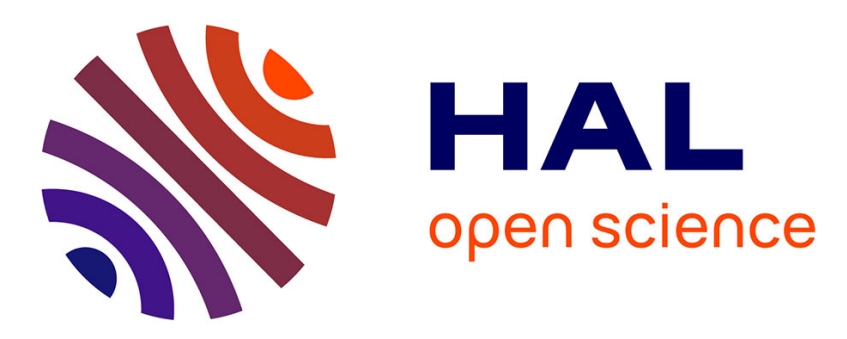

\title{
Incidence des temps et revenus professionnels sur le partage de l'activité domestique au sein de couples new-yorkais, tokyoïtes et parisiens
}

\author{
Arnaud Dupray, Anne-Marie Daune-Richard, Hiroatsu Nohara
}

\section{To cite this version:}

Arnaud Dupray, Anne-Marie Daune-Richard, Hiroatsu Nohara. Incidence des temps et revenus professionnels sur le partage de l'activité domestique au sein de couples new-yorkais, tokyoïtes et parisiens. Cahiers de recherche sociologique, 2017, 63, pp.43-66. 10.7202/1055718ar . halshs-02924360

\section{HAL Id: halshs-02924360 \\ https://shs.hal.science/halshs-02924360}

Submitted on 28 Aug 2020

HAL is a multi-disciplinary open access archive for the deposit and dissemination of scientific research documents, whether they are published or not. The documents may come from teaching and research institutions in France or abroad, or from public or private research centers.
L'archive ouverte pluridisciplinaire HAL, est destinée au dépôt et à la diffusion de documents scientifiques de niveau recherche, publiés ou non, émanant des établissements d'enseignement et de recherche français ou étrangers, des laboratoires publics ou privés. 
Modalités de partage de l'activité domestique au sein de couples new-yorkais, tokyoïtes et parisiens ${ }^{1}$

Arnaud Dupray, Anne-Marie Daune Richard, Hiroatsu Nohara

\section{Coordonnées des auteurs :}

Arnaud Dupray : Chargé d'Etudes au Céreq et chercheur associé au LEST, UMR7317.

Céreq, 10 place de la Joliette, BP 2132113567 Marseille Cedex 02.Email : dupray@cereq.fr Anne-Marie Daune-Richard: Chercheuse associée au LEST, UMR 7317. Lest, 35 Avenue Jules Ferry, 13626 Aix en Provence Cédex. Email : anne-marie.daune-richard@univ-amu.fr Hiroatsu Nohara: Chercheur associé au LEST, Lest; Yamanashigakuin University, 35 Avenue Jules Ferry, 13626 Aix en Provence Cédex. Email : hiroatsu.nohara@univ-amu.fr

\section{Cet article est paru:}

Dupray, A., Daune Richard, A.-M. \& Nohara, H. (2017). Incidence des temps etrevenus professionnels sur le partage de l'activité domestique au sein decouples new-yorkais, tokyoïtes et parisiens. Cahiers de recherche sociologique,(63), 43-66. https://doi.org/10.7202/1055718ar

\footnotetext{
${ }^{1}$ Cet article est issu d'un projet de recherche «NewDynam », co-financé par l'ANR (Agence nationale de la recherche) et JSPS (Japan Society for the Promotion of Science) sur les nouvelles dynamiques de l'emploi en France et au Japon. Les auteurs remercient Rengo-Soken pour la mise à disposition des données.
} 


\section{Modalités de partage de l'activité domestique au sein de couples new-yorkais, tokyoïtes et parisiens.}

\section{Introduction}

Bien que relative au domaine privé la répartition des activités domestiques dans le couple a des répercussions sur le marché du travail en contribuant aux inégalités professionnelles entre hommes et femmes. Le temps et sa distribution dans les différents pôles d'activité qui composent une existence est ainsi au cœur d'arbitrages qui touchent les ménages, la cellule familiale et, en même temps, la société dans sa gouvernance, à travers les leviers politiques qu'elle se donne pour organiser l'articulation entre les différents temps de vie. La répartition du temps de l'activité domestique entre conjoints est ainsi susceptible de refléter à la fois les inégales opportunités d'emploi et de carrière entre hommes et femmes et les processus sociétaux de légitimation et d'assignation qui encadrent les délibérations au sein du couple.

Cette question est abordée ici pour des métropoles appartenant à des économies de marché aux niveaux de développement comparables, les Etats-Unis, la France et le Japon, mais diversement outillées en dispositifs visant à concilier les vies professionnelle et familiale. Le propos est d'interroger d'une part, comment l'influence des déterminants de niveau individuel comme l'apport de chaque conjoint au revenu du ménage ou le temps passé à exercer une activité professionnelle sont susceptibles de varier en fonction du contexte sociétal du pays d'appartenance. D'autre part, la proximité de la répartition de l'activité domestique entre les couples new-yorkais, tokyoïtes et parisiens enquêtés et leur correspondance avec le contexte institutionnel et sociétal, est interrogée. Par rapport aux modèles issus de la théorie du capital humain, la position épistémologique adoptée ici considère que les individus, tout en agissant comme des acteurs rationnels, sont fortement imprégnés des contraintes institutionnelles et culturelles qu'ils intègrent dans leurs stratégies. La rationalité doit ainsi être comprise comme « située » dans un contexte qui encadre l'espace des décisions possibles.

L'examen, dans un premier temps, des contextes sociétaux à partir des grilles d'EspingAndersen et de Lewis dans lesquels évoluent ces couples urbains nous amèneront à formuler quatre hypothèses. Données et méthodologie seront présentées dans une deuxième section. Nous nous attacherons à étudier les modalités de partage des temps domestiques entre conjoints dans la partie Résultats puis à cerner en quoi et comment ces modalités varient d'une métropole à l'autre. 


\section{Les contextes sociétaux au regard du partage du travail rémunéré et non rémunéré}

Le partage du travail non rémunéré est une composante de la division sexuelle du travail car partout, à des degrés divers, le travail domestique et parental est plus assumé par les femmes que par les hommes tandis que le marché du travail demeure plus investi par ces derniers. Audelà de cette tendance générale, on constate des variations importantes d'un pays à l'autre des temps consacrés au travail domestique et au travail rémunéré (OCDE, 2012) ${ }^{2}$ ainsi que des contextes institutionnels plus ou moins favorables à leur égalisation selon le sexe.

La typologie élaborée par Esping-Andersen $(1990)^{3}$ permet de positionner les trois sociétés ${ }^{4}$ dans des espaces caractérisés par des modes distincts de régulation entre le marché du travail et les politiques sociales. En suivant l'indicateur du degré de « démarchandisation » - c'est-à-dire de non dépendance des individus par rapport au marché pour vivre - ${ }^{5}$ que ces régulations ouvrent, l'auteur éclaire leur influence sur la division sexuée des rôles domestique et professionnel.

Cependant, les recherches féministes ont montré l'importance de prendre en compte la division inégale et sexuée du travail rémunéré et non rémunéré et d'intégrer pleinement la famille dans l'analyse des Etats-providence. La démarchandisation est en effet, pour les femmes, fortement structurée par leur prise en charge du care (Lewis, 1992 ; Orloff, 1993) ${ }^{6}$. Dans sa forme pure, le modèle de «monsieur gagne-pain »n'a jamais existé : il a connu des interprétations différentes selon les histoires nationales. Jane Lewis (1992) analyse trois conjugaisons de ce modèle : «fort » représenté par l'Angleterre ; «faible » représenté par la Suède qui se rapproche d'un modèle familial à deux apporteurs et enfin un cas hybride, «modified» dans les termes de l'auteur représenté par la France, combinant promotion de l'indépendance économique des femmes et soutien à la famille en tant qu'institution.

\footnotetext{
${ }^{2}$ OCDE, 2012, Inégalités Hommes-Femmes. Il est temps d'agir, Editions OCDE.

${ }^{3}$ Esping-Andersen Gösta, (1999), Les trois mondes de l'Etat-providence. Essai sur le capitalisme moderne, Paris, PUF, 310 p. (1ère édition, en anglais: 1990).

${ }^{4}$ Alors que l'utilisation de références macro-institutionnelles et culturelles présuppose un cadre national, la limitation géographique de la comparaison ne peut prétendre au reflet des cadres sociétaux «représentatifs». Cependant, New York, Paris et Tokyo, non seulement par la taille de leur population mais aussi par leur force symbolique, permettent de concevoir une référence sociétale plus avancée en ce qui concerne les différents régimes d'Etat-providence.

${ }_{5}^{5}$ On reprend ici la traduction française de "decommodification" généralement utilisée et qu'Esping-Andersen définit ainsi : «La "démarchandisation" intervient lorsqu'un service est obtenu comme un dû et lorsqu'une personne peut conserver ses moyens d'existence sans dépendre du marché » $(1999$, p. 35)

${ }^{6}$ Lewis Jane, (1992), « Gender and the development of welfare regimes », Journal of European Social Policy, 3 , p. 159-171. Orloff Ann Shola, (1993), «Gender and the social rights of citizenship. State, policies and gender relations in comparative research », American Sociological Review, 58, p. 303-328.
} 
Dans la typologie de Lewis, le Japon pourrait être considéré comme une forme familiale achevée de «male breadwinner model » tandis que les Etats-Unis, compte tenu de la logique libérale et individualiste au cœur de la philosophie sociale américaine, devraient tendre vers un modèle «faible », c'est-à-dire de soutien à l'égalité des individus - femmes et hommes dans la société en général et la famille en particulier - mais sans l'aide d'un Etat-providence fort. De son côté la France est décrite comme mixant des traits appartenant au modèle de « monsieur gagne-pain » et d'autres la rapprochant d'un modèle « à deux apporteurs ».

Combiner ces deux typologies d'Etat-providence (Esping-Andersen) et de modèles familiaux (Lewis) permet de situer les emplois du temps des couples dans des contextes sociétaux distincts.

D'un côté, le Japon et la France s'inscrivent dans des régimes «conservateurs » d'Etatprovidence, régime qui, entre autres caractéristiques, tend à soutenir le statut de la famille comme unité privée de base de la société et à préserver des attitudes traditionnelles en matière de répartition des rôles familial et professionnel. Cependant, si l'institution familiale y constitue un référentiel des politiques publiques, ces deux pays ne partagent ni le même idéal de famille, ni les mêmes principes en matière de politique familiale, ni le même type de régulation du travail.

Le Japon se caractérise par un fort «familialisme» traditionnel ayant trois composantes principales : les entreprises ont en quelque sorte intégré la dimension familiale par l'emploi à vie et par la progression des salaires -masculins- qui reflètent l'évolution des charges familiales selon le cycle de vie (Nohara, 1999) ${ }^{7}$. il se double d'une faible intervention publique dans les affaires familiales, malgré les efforts entrepris depuis le milieu des années 90 avec le «Childcare leave law», contribuant à limiter l'essor de véritables carrières professionnelles pour les femmes (Imada, Ikeda, 2007) ${ }^{8}$. L'existence d'un marché du travail segmenté entre les emplois réguliers protégés et une diversité d'emplois atypiques ainsi qu'une culture organisationnelle masculine des grandes entreprises maintiennent la ségrégation horizontale et verticale des emplois (Nemoto, 2013) ${ }^{9}$.

La France se démarque par un «familialisme républicain » où l'Etat s'investit dans les politiques en faveur de la famille, en même temps qu'il soutient la femme en tant que travailleuse et favorise la conciliation travail-famille. En effet, dans sa philosophie sociale, la

\footnotetext{
${ }^{7}$ Nohara Hiroatsu, (1999), «L'analyse sociétale des rapports entre activités féminine et masculine. Comparaison France-Japon », Revue Française de Sociologie, 40-3, p. 531-558.

${ }^{8}$ Imada Sachiko, Ikeda Shingou, (2007), "The problems of the women's job continuity and the childcare leave system”, Japan Labor Review, 4 (2), p. 139-160.

${ }_{9}^{9}$ Nemoto Kumiko, (2013), "When culture resists progress: masculine organizational culture and its impacts on the vertical segregation of women in Japanese companies", Work, Employment and Society, 27(1), p. 153-169.
} 
France est prise dans une tension entre deux héritages (Commaille, 2001) ${ }^{10}$ : d'un côté, une vision de la famille constitutive de l'ordre social et politique, héritée de l'Ancien Régime ; de l'autre, une conception du lien social, héritée de la philosophie des Lumières et de la Révolution, où l'individu est une référence centrale. Dans cette tension s'est développée une tradition de « choix », pour les femmes, de s'inscrire ou non sur le marché du travail grâce à divers dispositifs publics comme la prestation d'accueil du jeune enfant (PAJE).

Dans la typologie d'Esping-Andersen, les Etats-Unis font figure de cas-type du régime « libéral » d'Etat-providence caractérisé en particulier par une intervention étatique limitée dans les affaires familiales et par la faiblesse de l'offre publique de services sociaux (Morgan, 2013). Si le modèle de «monsieur gagne-pain » a été prégnant dans les années 50 - au moins dans les couches sociales moyennes et aisées - il laisse la place aujourd'hui à un modèle où chaque conjoint contribue aux ressources monétaires de la famille, permettant le recours éventuel au marché pour pallier le déficit de services publics (externalisation du care).

Les comparaisons internationales sur de nombreux pays ont pu mettre en évidence l'influence de ces variables de contexte national sur la répartition du temps domestique au sein des couples (Davis et Greenstein, 2004 ; Fuwa, 2004 ; Geist, 2005 ; Hook, 2010 ${ }^{11}$ ). Une part importante de la variation internationale des temps d'activité domestique et de modalités de partage résulte ainsi des contextes sociétaux en matière de politique sociale, de capacité des systèmes nationaux à promouvoir l'égalité entre hommes et femmes dans l'emploi mais aussi de l'adhésion générale de la population à une vision égalitaire - ou non - des rôles sexués.

Les contextes nationaux esquissés ci-dessus permettent d'avancer des hypothèses sur la division de l'activité domestique au sein des ménages.

$\mathrm{Au}$ Japon, les freins auxquels sont confrontées les femmes sur le marché du travail et l'insuffisance de structures de garde de jeunes enfants tendent à les cantonner dans la gestion de la sphère privée tendant à préserver la logique du male breadwinner. Dans la logique libérale et égalitariste qui prévaut aux Etats-Unis, l'égalité se construit sur le marché et en quelque sorte se passe d'un soutien institutionnel à la famille. L'externalisation des activités domestiques et de care est donc renvoyée pour l'essentiel au marché et aux politiques

\footnotetext{
${ }^{10}$ Commaille Jacques, (2001), Les injonctions contradictoires des politiques publiques à l'égard des femmes, in Laufer Jacqueline, Maruani Margaret, Marry Catherine (dir.), Masculin-féminin : questions pour les sciences de l'homme, PUF, p.129-148.

${ }^{11}$ Davis Shannon N., Greenstein Theodore N., (2004), "Cross-national variations in the division of household labor", Journal of Marriage and Family, 66, p. 1260-1271. Fuwa Makiko, (2004), « Macro-level gender inequality and the division of household labor in 22 countries », American Sociological Review, 69, p. 751-767. Geist Claudia, (2005), "The welfare State and the home: regime differences in the division of labour". European Sociological Review, 21(1), p.23-41. Hook Jennifer L. (2010), "Gender Inequality in the Welfare State: Sex Segregation in Housework”, 1965-20031, American Journal of Sociology, 115(5), p. 1480-1523.
} 
d'entreprises. Mais, de ce fait, il existe une forte symétrie dans les attitudes des acteurs institutionnels et professionnels vis-à-vis des hommes et des femmes depuis les lois antidiscriminatoires des années 60 et 70 . La France se signale par une configuration hybride, avec une dissymétrie entre une politique d'égalité sur le marché du travail instrumentée par des lois successives depuis les années 1970 et une politique familiale généreuse mais dont les effets s'exercent parfois à l'encontre du premier objectif (Périvier, 2010) ${ }^{12}$.

De là en découle l'hypothèse $1:$ le partage de l'activité domestique dans le couple devrait être le mois inégalitaire à New-York et le plus inégalitaire à Tokyo, les couples parisiens se situant entre les deux.

Au regard du niveau microéconomique de l'arbitrage, suivant Becker (1985) ${ }^{13}$ chacun devrait se spécialiser selon ses avantages comparatifs, l'homme dans les activités professionnelles si celles-ci sont plus rémunératrices que pour les femmes. Il en résulte que le pouvoir de négociation de la femme et sa capacité à imposer un partage plus égalitaire des tâches résulterait de sa contribution au revenu du ménage selon la théorie du «bargainingexchange » (Brines, 1994) ${ }^{14}$. Or, cet effet devrait être d'autant plus sensible dans un pays conservateur où la séparation des rôles sociaux entre hommes et femmes demeure marquée.

D'où 1 'hypothèse 2 : On s'attend à ce que la contribution des femmes au revenu du ménage influence davantage la répartition du travail domestique au sein du couple dans un régime conservateur que dans un régime libéral qui promeut davantage l'égalité d'accès aux ressources économiques entre hommes et femmes.

Suivant la perspective d'un temps plus ou moins contraint pour participer aux tâches domestiques, il convient de prendre en compte l'impact des temps professionnels respectifs des conjoints pour évaluer leurs participations aux tâches domestiques mais aussi l'organisation du leur temps de travail, plus ou moins régulière et standardisée (Presser, 1994 $)^{15}$. Et on s'attend, là aussi, à ce que cette substituabilité entre temps disponibles des conjoints soit plus sensible, et donc plus significatif l'effet des temps d'activités professionnels sur la répartition du temps domestique entre les conjoints, dans les pays où une répartition traditionnelle des rôles sociaux persiste dans les représentations.

\footnotetext{
12 Périvier Hélène, (2010), «La logique sexuée de la réciprocité dans l'aide sociale », Revue de l'OFCE, 114, p. 237-263.

13 Becker, Gary S, (1985), «Human capital, effort and the sexual division of labor », Journal of Labor Economics, 3(1-pt.2), p. 33-58.

${ }^{14}$ Brines Julie, (1994), «Economic dependency, gender and the division of labor at home », American Journal of Sociology, 100(3), p. 652-688.

${ }^{15}$ Presser, H. B. (1994). Employment schedules among dual-earner spouses and the division of household labor by gender. American Sociological Review, 59(3), p. 348-364.
} 
Il en résulte l'hypothèse 3: L'influence sur le partage des activités domestiques du temps professionnel et type d'organisation du travail rémunéré de chaque conjoint est supposée plus importante dans les régimes conservateurs d'Etat-providence que dans un régime libéral.

Enfin, hypothèse 4, considérant les régimes d'Etat-providence, les modèles de partage du travail domestique devraient être plus proches entre couples parisiens et tokyoïtes qu'entre ces couples et les new-yorkais. Ces derniers sont en revanche susceptibles d'être plus proches des couples français en ce qui concerne leurs caractéristiques éducatives et professionnelles, le modèle familial de « deux contributeurs à temps plein» constituant le modèle dominant.

\section{Données et méthodologie}

Avant de présenter les mesures obtenues dans les trois métropoles, un détour par les données et les méthodes de traitement s'impose.

\section{Les données}

Nous exploitons les données issues d'une enquête de comparaison internationale sur les budget-temps réalisée en novembre 2007 dans les trois pays à l'initiative de l'institut de recherche «Rengo soken» qui est rattaché à la Confédération syndicale japonaise «Rengo $»^{16}$. Cette enquête concernait 400, 412 et 418 couples résidant respectivement à New York, Paris et Tokyo ou dans leurs environs. Chaque conjoint étant interrogé séparément, elle a permis la collecte d'environ 800 questionnaires appariés par couple et par pays, offrant un matériau statistique unique pour l'analyse de l'organisation des temps domestiques et professionnels.

Le champ de l'enquête est restreint, pour mieux assurer la comparabilité. D'une part, l'enquête cible les couples dont les conjoints - le statut marital n'étant pas renseigné - sont âgés de plus de 20 ans et de moins de 55 ans, et dont au moins l'un d'eux est économiquement «actif». D'autre part, elle se limite aux «classes moyennes et supérieures citadines» dont témoignent les niveaux d'études des enquêtés avec près de $70 \%$ des hommes et les trois quart des répondantes qui sont, dans les trois échantillons, diplômés de l'enseignement supérieur. Bien que ces catégories ne renvoient pas forcément aux mêmes réalités sociologiques d'un pays à l'autre, il est clair que les individus enquêtés ont des attributs socio-économiques plus favorables et se caractérisent par des normes de comportements sociaux sans doute moins «traditionalistes» que les populations nationales dont ils émanent.

\footnotetext{
${ }^{16}$ Rengo-soken, 2009, « Seikatsujikann no kokusaichosa ; Enquête de comparaison internationale sur l'usage du temps », 322 page, Tokyo.
} 
L'enquête administrée par internet a été réalisée, dans les trois dernières semaines du mois de novembre 2007, par IPSOS aux Etats-Unis et en France, et par le Japan Research Center au Japon. Elle a obtenu des taux de réponse de 55 à $71 \%$ selon le pays. Les informations collectées concernent avant tout les temps passés dans différents types de tâches: domestiques, de soin aux enfants ou rémunérées. Enfin, l'emploi occupé est décrit au travers de dimensions classiques comme la taille de l'entreprise, le secteur, la catégorie socioprofessionnelle et le revenu.

Différentes mesures du temps d'activité domestique sont disponibles dans l'enquête. Elles diffèrent en fonction de leur périmètre et de l'unité temporelle prise en compte (un jour de semaine, un jour de week-end ou la semaine entière). Nous avons choisi de nous concentrer sur les activités domestiques les plus quotidiennes et routinières - les courses, le ménage et la préparation des repas - et d'écarter les activités qui pourraient davantage se rapprocher d'un hobby : activités liées au jardinage, à l'entretien de la voiture ou au bricolage. Par ailleurs, en accord avec nombre de résultats publiés sur le sujet, nous privilégions une échelle de mesure correspondant à la semaine entière ${ }^{17}$.

\section{Méthode}

Les mesures utilisées du temps d'activité domestique sont de deux types, en valeur et en pourcentage. Pour analyser les facteurs pesant sur la répartition du temps d'activité domestique au sein des couples, on calcule la proportion du temps total assuré par les deux conjoints, que la femme prend en charge. L'intérêt d'une telle mesure est de pouvoir mieux capter les dimensions de justice distributive et d'équité dans la répartition du travail domestique (Greenstein, 2000) ${ }^{18}$.

Deux spécifications principales des modèles ont été appliquées par pays, l'une tenant compte de la configuration de couple en fonction du rapport de chacun des conjoints au marché du travail (modèle 1), l'autre intégrant directement les variables de durée et de régime de temps de travail professionnel sur des effectifs restreints alors aux couples dont l'homme et la femme exercent une activité rémunérée (modèle 2). Dans tous les cas, l'ambition des modèles est avant tout descriptive. Les interprétations ont été consolidées en testant plusieurs spécifications des variables d'éducation, de revenu et de temps de travail rémunéré.

\footnotetext{
${ }^{17}$ Bien que relatifs à des populations spécifiques en termes de zone géographique et de catégorie sociale, les temps mesurés ici sont cohérents avec les données issues des enquêtes internationales ou nationales de grande ampleur comme l'American Time Use Survey (ATUS) de 2011, l'enquête Emploi du Temps 2010 pour la France ou le National Survey on Family and Economic Conditions (NSFEC) de 2009 pour le Japon.

${ }^{18}$ Greenstein Theodore N., 2000, «Economic Dependence, Gender and the division of labor in the home: a replication and extension », Journal of Marriage and Family, 62, p. 322-335.
} 
On procède par des régressions linéaires estimées avec une matrice de variance-covariance robuste à l'hétéroscedasticité. ${ }^{19}$

Sur la base des résultats des régressions, on appellera «modèle de partage » les effets moyens propres à chaque population conditionnellement aux variables incluses dans les modèles. Ceci nous permettra de faire des simulations en fixant soit un modèle de partage propre à une des trois populations, soit les caractéristiques des couples vivant dans une des métropoles.

\section{Résultats}

\section{Quelles répartitions du travail domestique?}

Le temps d'activité domestique total, en additionnant le temps déclaré par chacun des conjoints, est le plus élevé à New York avec près de 25 heures par semaine en moyenne, suivi par Tokyo avec un peu moins de 21 heures et de Paris avec environ 17h45 (Tableau 1). La répartition est la plus inégalitaire à Tokyo où les femmes y consacrent une durée plus de cinq fois supérieure à celle de leur conjoint puisque $82 \%$ du volume total leur incombe. A Paris et New York, la proportion à la charge des femmes est en moyenne respectivement de 72 et $64 \%$. Ces résultats confirment l'hypothèse 1 .

Reflets de ces situations de partage plus ou moins inégalitaires, c'est à Tokyo que la proportion de femmes qui se disent satisfaites de la division du travail domestique avec leur partenaire est la plus modeste : $46 \%$ contre $55 \%$ et $77 \%$ respectivement dans les métropoles parisienne et new yorkaise. Le partage apparaîtrait encore plus défavorable à l'aune d'un jour ouvrable, notamment à Tokyo où les hommes limitent à environ 17 minutes leur disponibilité quotidienne pour ces tâches contre 56 minutes à New York et près de la demi-heure à Paris. Le week-end est donc l'occasion pour les hommes de relever leur investissement domestique, notamment à Tokyo et à Paris, et à un moindre degré à New York, en cohérence pour ces derniers, avec une participation aux tâches domestiques plus équilibrée au quotidien.

Ces écarts sont susceptibles de masquer une variété de répartition des couples enquêtés au regard de leur participation au marché du travail et de leur temps effectif consacré à une activité professionnelle, conditionnant ainsi la participation de chacun aux tâches domestiques.

A cet effet, cinq configurations de couple ont été distinguées selon la place de la femme vis-àvis de l'emploi. La catégorie 1 rassemble les couples dont les deux conjoints occupent un

19 Des tests Durbin-Wu-Hausman d'endogénéité des variables de temps de travail et de revenus dans les régressions ne permettent pas de rejeter l'hypothèse d'exogénéité de ces dimensions. 
emploi régulier à plein temps (tableau 1). Les catégories 2, 3 et 4 concernent des couples où l'homme est toujours employé à temps plein mais où le statut d'emploi de la femme change : respectivement, en emploi atypique (intérim, contrat temporaire...) et travaillant moins de 30 heures par semaine, en emploi atypique mais d'au moins 30 heures hebdomadaires, enfin inactive ou au chômage pour la catégorie 4 . La catégorie 5 constitue un groupe résiduel qui réunit tous les couples où l'homme n'exerce pas à plein temps dans un statut d'emploi stable quel que soit le statut de sa compagne. La distribution de ces configurations varie selon la métropole : Tokyo se signale par le faible poids relatif de la catégorie 1 comparativement à Paris et New York où elle est majoritaire, représentant respectivement environ $67 \%$ et $56 \%$ des couples échantillonnés. A Tokyo, la situation où la femme occupe un emploi atypique à temps partiel est quasiment aussi courante que la première (près de $28 \%$ ), alors qu'elle ne concerne qu'environ $10 \%$ des couples dans les deux autres métropoles. Corrélativement, c'est à Tokyo que l'on trouve la plus forte proportion de femmes occupant un emploi atypique quelle que soit sa durée (catégories 2 et 3 ) et de couples où l'homme est seul pourvoyeur des revenus du travail (catégorie 4, $19 \%$ ).

La distribution de ces configurations d'une métropole à l'autre contribue à rendre compte des mesures de partage observées parmi les couples parisiens et tokyoïtes puisque les temps absolu et relatif de travail domestique augmentent significativement pour les femmes qui travaillent à temps partiel ou sont au foyer. A New York, même si on observe un accroissement du temps absolu, l'inégalité du partage ne baisse que très faiblement quand on passe d'une situation de travail à temps plein à une situation d'inactivité pour la femme (ou de chômage $)^{20}$, en passant par le temps partiel. Tout se passe comme s'il existait une plus forte indépendance du temps d'activité domestique - et de son partage - par rapport aux temps professionnels des conjoints dans les couples new yorkais, comparativement aux couples des deux autres métropoles. A Paris et plus encore à Tokyo, le temps domestique est plutôt désinvesti (réinvesti) par les femmes à mesure que leur temps professionnel s'accroît (se contracte) : le temps partiel «tire» ainsi ces japonaises et ces françaises vers un régime d'activité domestique se rapprochant dans sa durée de celui des femmes inactives.

Ainsi, à configuration de couple donnée, on retrouve la division la plus traditionnelle à Tokyo et la plus équilibrée à New York. La présence d'enfants en bas âge ou pré-adolescents, ne modifie que très marginalement les investissements respectifs de l'homme et de la femme et

\footnotetext{
${ }^{20}$ La catégorie des couples où seul l'homme pourvoit aux ressources du ménage inclut les situations où la femme est en recherche d'emploi. Or, cette fraction représente 5,5\% des ménages dans l'échantillon américain contre 1 et 2\%, respectivement au Japon et en France. Rappelons que l'enquête s'est déroulée en 2007, avant la crise financière.
} 
plutôt dans le sens d'un renforcement du déséquilibre comme on pouvait s'y attendre (Craig \& Mullan, 2010) $)^{21}$.

Au total, cette comparaison dessine bien un modèle japonais familialiste, comparativement à la France et aux Etats-Unis où l'emploi de l'épouse fait moins office de facteur d'ajustement et où les activités domestiques sont un peu mieux partagées, surtout parmi les couples NewYorkais.

En définitive, une fois pris en compte le temps d'activité professionnelle et la répartition des femmes en couple selon leur statut à l'égard de l'emploi, ce premier examen tend à montrer que la restriction de la place des femmes japonaises dans l'emploi rémunéré apparaît comme une condition de leur surinvestissement dans les activités domestiques et ce, d'autant plus que leurs conjoints sont plus massivement qu'ailleurs accaparés par leur activité professionnelle le temps total passé hors de leur domicile pour leur activité professionnelle s'établit à environ 65 heures par semaine pour les Tokyoïtes appartenant aux quatre premières configurations de couples $^{22}$. L'incidence de l'investissement professionnel des femmes sur leur contribution à l'activité domestique suggère une différence de degré plus que de nature dans les modes d'arbitrage entre conjoints dans les métropoles française et japonaise, tandis que les couples new yorkais se signalent par un cloisonnement plus affirmé entre activité professionnelle et activité domestique de chacun des partenaires : l'investissement des hommes dans les activités domestiques tend en effet à s'accroître avec celui de leur compagne, indépendamment de leur charge professionnelle.

\footnotetext{
${ }^{21}$ Craig L., Mullan K. (2010) "Parenthood, gender and work-family time in the united States, Australia, Italy, France and Denmark", Journal of Marriage and Family, 72, p. 1344-1361.

22 L'une des raisons de ces longues durées de travail est liée à la pratique très répandue des heures supplémentaires. Il existe en effet un large accord des salariés et employeurs autour de leur usage : les entreprises y recourent afin de conserver une flexibilité productive, et une grande partie des employés y consentent pour augmenter leur pouvoir d'achat (Nohara, 1999).
} 
Tableau 1 : Temps d'activité domestique, absolu et relatif, selon la configuration de couple

\begin{tabular}{|c|c|c|c|c|c|c|c|c|c|c|c|c|}
\hline \multirow{3}{*}{ Metropole } & \multicolumn{5}{|c|}{ New York } & \multicolumn{4}{|c|}{ Paris } & \multicolumn{3}{|c|}{ Tokyo } \\
\hline & & \multicolumn{2}{|c|}{$\begin{array}{l}\text { Temps } \\
\text { consacré au } \\
\text { travail } \\
\text { domestique } \\
\text { par semaine } \\
\text { en heures (a) }\end{array}$} & \multicolumn{2}{|c|}{$\begin{array}{l}\% \text { effectué par } \\
\text { la femme }\end{array}$} & \multicolumn{2}{|c|}{$\begin{array}{l}\text { Temps } \\
\text { consacré au } \\
\text { travail } \\
\text { domestique } \\
\text { par semaine } \\
\text { en heures (a) }\end{array}$} & \multicolumn{2}{|c|}{$\begin{array}{l}\% \text { effectué par } \\
\text { la femme }\end{array}$} & \multicolumn{2}{|c|}{$\begin{array}{l}\text { Temps } \\
\text { consacré au } \\
\text { travail } \\
\text { domestique } \\
\text { par semaine } \\
\text { en heures (a) }\end{array}$} & \multirow[t]{2}{*}{$\begin{array}{l}\% \text { effectué par } \\
\text { la femme }\end{array}$} \\
\hline & $\begin{array}{l}\mathrm{N} \\
\% \\
\end{array}$ & F & $\mathrm{H}$ & & $\begin{array}{l}\mathrm{N} \\
\% \\
\end{array}$ & $\mathrm{~F}$ & $\mathrm{H}$ & & $\begin{array}{l}\mathrm{N} \\
\% \\
\end{array}$ & $\mathrm{~F}$ & $\mathrm{H}$ & \\
\hline $\begin{array}{l}\text { Catégorie 1: } \\
\text { Les } 2 \text { conjoints en emploi } \\
\text { régulier à plein temps } \\
\end{array}$ & $\begin{array}{l}223 \\
55,8 \\
\end{array}$ & 14,7 & 7,5 & 67,2 & $\begin{array}{r}274 \\
66,5 \\
\end{array}$ & 11,5 & 5 & 70,8 & $\begin{array}{r}118 \\
28,2 \\
\end{array}$ & 13,4 & 4 & 75 \\
\hline $\begin{array}{l}\text { Catégorie } 2: \\
\text { H. à plein temps et } \mathrm{F} \text {. en } \\
\text { emploi atypique }<30 \mathrm{~h}\end{array}$ & $\begin{array}{l}42 \\
10,5 \\
\end{array}$ & 18,5 & 8,1 & 64,4 & $\begin{array}{r}40 \\
9,7 \\
\end{array}$ & 15,7 & 3 & 82,6 & $\begin{array}{r}115 \\
27,6 \\
\end{array}$ & 20 & 2,2 & 89,8 \\
\hline $\begin{array}{l}\text { Catégorie } 3 \text { : } \\
\text { H. à plein temps et } \mathrm{F} \text {. en } \\
\text { emploi atypique } 30 \mathrm{~h} \text { et }+ \\
\end{array}$ & $\begin{array}{l}15 \\
3,7\end{array}$ & 17,6 & 9,4 & 67 & $\begin{array}{l}25 \\
6,1\end{array}$ & 11,7 & 7,3 & 63,5 & $\begin{array}{l}31 \\
7,4\end{array}$ & 15,1 & 3,3 & 79,8 \\
\hline $\begin{array}{l}\text { Catégorie } 4 \text { : } \\
\text { H. à plein temps et } F \text {. } \\
\text { inactive ou au chômage. }\end{array}$ & $\begin{array}{l}63 \\
15,8 \\
\end{array}$ & 21,5 & 10,6 & 63,7 & $\begin{array}{r}31 \\
7,5 \\
\end{array}$ & 20,6 & 3,9 & 82,8 & $\begin{array}{r}80 \\
19,1 \\
\end{array}$ & 23,6 & 3,1 & 86,9 \\
\hline $\begin{array}{l}\text { Catégorie } 5 \text { : } \\
\text { Autres (H. non à plein } \\
\text { temps) }\end{array}$ & $\begin{array}{l}57 \\
14,2 \\
\end{array}$ & 15,1 & 11,1 & 52 & $\begin{array}{r}42 \\
10,2 \\
\end{array}$ & 12,5 & 6,4 & 68,6 & $\begin{array}{r}74 \\
17,7 \\
\end{array}$ & 14,5 & 4 & 78,7 \\
\hline Ensemble des couples & 400 & 16,3 & 8,6 & $64 \%$ & 412 & 12,7 & 5,02 & $72 \%$ & 418 & 17,5 & 3,4 & $82 \%$ \\
\hline
\end{tabular}

(a): Les durées hebdomadaires conjuguent le temps passé aux tâches domestiques à la fois pendant les jours ouvrés et les jours de repos. 


\section{Les déterminants individuels et d'emploi de la répartition de l'activité domestique dans les} couples

On examine ici les facteurs susceptibles d'influencer le partage des activités domestiques, avec l'hypothèse d'effets variables des dimensions du revenu et du temps de travail professionnel selon le pays d'appartenance des couples.

Parmi les dimensions explicatives introduites dans les modèles (répertoriées au tableau 2), la moyenne de l'âge des deux conjoints figure la génération d'appartenance du couple (Brayfield, 1992) ${ }^{23}$. Elle se justifie selon l'idée que les attitudes égalitaires dans le partage des tâches domestiques se retrouvent plus fréquemment parmi les couples jeunes. Le nombre et l'âge des enfants peuvent modifier également le temps d'activité domestique, ceux de 12 ans au plus au sein du ménage sont ainsi pris en compte - âge de fin d'enseignement élémentaire au Japon et aux Etats-Unis ${ }^{24}$. Le revenu total du ménage en dehors des revenus du travail (revenus du capital et immobiliers, pension, allocations etc...) est pris en compte car il figure la capacité de pouvoir d'achat du couple pouvant permettre de reporter sur le marché une fraction des activités domestiques. Aucune information sur cette partie éventuellement externalisée n'est disponible dans l'enquête. En revanche, trois questions relatives au recours à une aide extérieure concernant le ménage, la préparation des repas et le repassage sont intégrées dans les modèles au même titre que la durée totale du travail domestique dans le ménage susceptible d'influencer sa répartition (Fuwa \& Cohen, 2007) ${ }^{25}$.

Introduire une indicatrice de revenu relatif de l'épouse par rapport à son mari permet de tester un effet de capacité de négociation. A cet effet, on recourt à l'indicateur proposé à l'origine par Sorensen et Mc Lanahan $(1987)^{26}$ qui est le taux de dépendance économique (TDE) de la femme par rapport à l'homme : $\mathrm{TDE}=\mathrm{W}_{\mathrm{f}}-\mathrm{W}_{\mathrm{h}} /\left(\mathrm{W}_{\mathrm{f}}+\mathrm{W}_{\mathrm{h}}\right)$ où $\mathrm{W}$ figure le salaire et l'indice, le sexe de la personne. Il varie de -1 si l'homme est seul contributeur à +1 dans le cas inverse formellement ici, cet indicateur figure le taux d'indépendance économique de la femme par rapport à son conjoint. Alternativement, on suivra Gupta (2006) ${ }^{27}$ en testant l'impact des revenus respectifs de l'homme et de la femme en lieu et place de TDE.

\footnotetext{
${ }^{23}$ Brayfield April A., (1992), "Employment resources and housework in Canada", Journal of Marriage and the Family, 54, p. 19-30.

${ }^{24}$ Ajoutons que le nombre moyen d'enfants par couple est assez homogène entre les trois échantillons variant de près de 0,8 à Tokyo à 1 en France en passant par 0,9 à New-York.

${ }^{25}$ Fuwa, Makiko, Cohen Philip N. (2007), "Housework and social policy." Social Science Research, 36, p. 512530.

${ }^{26}$ Sorensen Annemette, Mclanahan Sara, (1987), "Married women economic dependency”, American Journal of Sociology, 93(3), p. 659-687.

${ }^{27}$ Gupta Sanjiv, (2006), "Her money, her time: women's earnings and their housework hours", Social Science Research, 35, p. 975-999.
} 
Le niveau d'études de chacun des conjoints est intégré dans les modèles. En l'absence d'information sur les opinions individuelles sur les rôles de genre, un niveau d'éducation élevé refléterait une opinion progressiste en la matière : la propension de l'homme à assumer des tâches domestiques serait alors croissante avec son niveau d'éducation.

Pour le travail rémunéré, aux durées hebdomadaires de travail de chaque conjoint - lesquelles restreignent le temps disponible pour participer aux tâches ménagères - sont ajoutées des dichotomiques spécifiant les rythmes horaires du travail professionnel. Ils valent 1 si l'horaire de travail est flexible sans plage de présence obligatoire, en cas de travail posté ou d'une autre forme flexible d'horaire. Une personne travaillant avec de tels horaires est ainsi supposée moins disponible pour l'activité domestique.

Les constantes des modèles font apparaître qu'à mêmes caractéristiques individuelles, le partage des tâches domestiques varie d'une métropole à l'autre (Tableau 2).

Par rapport à un couple où le mari est en emploi stable à plein temps et où l'épouse travaille dans un emploi atypique moins de 30 heures par semaine, Paris et Tokyo se distinguent par une moindre charge domestique relative des femmes lorsqu'elles sont actives à plein temps conjointement à leur époux. A New York la variation du partage n'est pas significative entre les quatre premières configurations de couple même si, comme dans les autres métropoles, le partage est plus équilibré lorsque l'homme n'a pas de situation professionnelle régulière à plein temps.

La délégation d'un type de tâche domestique à un tiers n'apparaît jouer significativement en faveur d'un partage plus égalitaire que dans l'agglomération new yorkaise lorsque les couples délèguent la préparation des repas. Le nombre d'enfants pèse négativement sur la répartition du travail seulement dans l'agglomération parisienne, en cohérence avec des résultats antérieurs (Pailhé \& Solaz, 2009) ${ }^{28}$.

L'âge moyen du couple n'a pas d'effet quel que soit le pays, mais les couples japonais dont la femme a entre 30 et 40 ans présentent un partage plus équilibré que celles des générations antérieures (femmes de plus de 40 ans).

L'introduction simultanée des niveaux d'études des conjoints dans les régressions montre que seul le niveau d'études du mari interfère avec la valeur du partage de l'activité domestique. C'est particulièrement le cas parmi les couples new-yorkais où par rapport à des niveaux d'études équivalents au supérieur court, les sortants à des niveaux plus modestes

\footnotetext{
${ }^{28}$ Pailhé, Ariane, Solaz Anne, (2009), "Les ajustements professionnels des couples autour des naissances: une affaire de femmes?" In Ariane Pailhé and Anne Solaz (eds) Entre famille et travail: Des arrangements de couple aux pratiques des employeurs, Paris, La Découverte, p. 167-186.
} 
appartiennent à des couples où la division de l'activité est plus inégale, avec une charge accrue pour les épouses. Des effets de même sens ne sont sensibles que pour le niveau d'études le plus modeste (sortie au niveau secondaire avant la terminale) dans les couples parisiens et tokyoïtes.

A Tokyo, le revenu du travail des femmes leur permet d'alléger leur charge domestique au profit de leur mari améliorant de fait leur capacité à négocier leur temps conformément à une perspective de «bargaining-exchange ». A l'inverse, le revenu apporté par le mari tend à élever la charge domestique de sa compagne, mais dans une moindre mesure. L'indépendance économique de l'épouse affecte aussi le partage dans les couples parisiens mais avec une intensité moindre qu'à Tokyo - résultat cohérent avec ceux de Sofer et Rivazi $(2009)^{29}$. L'absence d'effet pour les couples new yorkais (du taux de dépendance économique comme des revenus individuels) s'éclaire si le taux est introduit en quatre catégories dans le modèle : $[-1 ;-0,5[,[-0,5 ;-0,1[,[-0,1 ; 0,05[,[0,05 ;+1]$.

Au-delà d'un taux de $-0,5$, les coefficients des tranches supérieures manifestent des réductions de la participation des femmes américaines aux tâches domestiques qui restent d'ampleurs comparables - un test du Wald ne rejette pas l'égalité des trois coefficients. L'effet n'est donc sensible qu'avec les premiers dollars gagnés - résultat corroborant ceux de Killewald et Gough $(2010)^{30}$ - donnant aux femmes américaines l'opportunité de desserrer la dépendance économique et de déléguer les tâches domestiques les plus faciles à externaliser comme le ménage.

L'hypothèse d'un pouvoir de négociation qui s'accroît avec la participation de la femme aux ressources du ménage semble ainsi confirmée pour les couples japonais et à un degré moindre pour les français, conformément à l'hypothèse 2 .

Ceci laisse alors présumer une substituabilité partielle entre les investissements domestiques des conjoints. Le pouvoir de négociation, représenté ici par le taux de dépendance économique, montre ainsi une incidence plus importante sur le partage dans un régime conservateur d'Etat-providence que dans un régime libéral où les attitudes égalitaires des employeurs et des institutions vis-à-vis des aspirations professionnelles des hommes et des femmes neutraliseraient, pour ces couples socio-économiquement favorisés, l'influence du pouvoir économique.

\footnotetext{
${ }^{29}$ Sofer, Catherine, and Sayyid Salman Rizavi. (2009), "Women's relative position and the division of household work: A study of French couples." European Journal of Economic and Social Systems, 22(2), p. 13-26.

${ }^{30}$ Killewald, A., Gough, M. (2010), “Money isn't everything: wives' earnings and housework time”, Social Science Research, 39, p. 987-1003.
} 
Tableau 2: Facteurs explicatifs de la part du travail domestique assurée par les femmes

\begin{tabular}{|c|c|c|c|c|c|c|}
\hline \multirow[b]{2}{*}{ Modèles } & \multicolumn{2}{|c|}{ New York } & \multicolumn{2}{|c|}{ Paris } & \multicolumn{2}{|c|}{ Tokyo } \\
\hline & (1) & $(2)$ & (1) & (2) & (1) & $(2)$ \\
\hline \multicolumn{7}{|l|}{ Type de couple: } \\
\hline Les 2 à plein temps & 5,651 & & $-7,103 * *$ & & $-6,819 * *$ & \\
\hline Femme inactive & $-4,442$ & & 2,121 & & $-4,520 *$ & \\
\hline Femme en emploi atypique $<30 \mathrm{~h}$ & Ref & & Ref & & Ref & \\
\hline Femme emploi atypique $>30 \mathrm{~h}$ & 5,611 & & $-13,33 * *$ & & $-5,276$ & \\
\hline résiduelle & $-9,755 *$ & & $-6,840$ & & $-6,125 *$ & \\
\hline \multicolumn{7}{|l|}{ Délégation de : } \\
\hline repassage & $-3,237$ & 1.319 & $-5,580$ & -4.399 & 5,289 & 9.565 \\
\hline ménage & 3,530 & 1.268 & $-3,339$ & -3.185 & 0,590 & 0.955 \\
\hline cuisine & $-11,94 * * *$ & $-11.65^{*}$ & 3,437 & -0.232 & $-8,739$ & -8.474 \\
\hline Famille étendue & 3,866 & 7.206 & $15,70 * *$ & 24.64 & 1,447 & 4.717 \\
\hline Age moyen du couple & $-0,331$ & -0.276 & 0,326 & 0.364 & $-0,237$ & -0.361 \\
\hline \multicolumn{7}{|l|}{ Age de la femme } \\
\hline$<30$ ans & $-4,623$ & -2.512 & 0,739 & 0.208 & $-3,373$ & -3.377 \\
\hline entre 30 et 40 ans & $-5,792$ & -3.849 & $-3,251$ & -4.004 & $-6,544^{*}$ & -6.733 \\
\hline Plus de 40 ans & Ref, & Ref. & Ref & Ref. & Ref. & Ref. \\
\hline $\begin{array}{l}\text { Nombre d'enfants } \\
\text { de moins de } 13 \text { ans }\end{array}$ & 0,299 & -0.299 & $1,837 *$ & 1.174 & 0,468 & 0.00932 \\
\hline $\begin{array}{l}\text { Niveau d'études de la femme : } \\
\text { Obligatoire jusqu'au } \\
\text { Secondaire- Bac }\end{array}$ & $-3,579$ & -5.991 & $-3,066$ & -2.855 & 0,0140 & -0.789 \\
\hline $\begin{array}{l}\text { Niveau Bac }+2 \text { ans } \\
\text { Bac }+4 \text { ans (Graduates) }\end{array}$ & Ref, & Ref. & Ref. & Ref. & Ref. & Ref. \\
\hline & $-1,788$ & -1.209 & $-0,209$ & -0.443 & 0,647 & -0.0996 \\
\hline $\begin{array}{l}\text { Au moins } 5 \text { ans post-Bac } \\
\text { (postgraduates) }\end{array}$ & $-5,483$ & -6.054 & $-2,500$ & -2.239 & $-5,200$ & -10.96 \\
\hline $\begin{array}{l}\text { Niveau d'études de l'homme: } \\
\text { Obligatoire (fin de } 3^{\text {ème }} \text { ) }\end{array}$ & & & & & & \\
\hline Cosondaim Do & $7,618 * *$ & $12.40 * *$ & $9,463 * * *$ & $10.26^{* *}$ & $14,13 * *$ & $11.41 * *$ \\
\hline & $14,02 * * *$ & $12.39 * *$ & 2,743 & 0.990 & 4,137 & 1.438 \\
\hline Niveau Bac +2 ans & & & & & & \\
\hline Bac +4 ans (Graduates) & Ref, & Ref. & Ref. & Ref. & Ref. & Ref. \\
\hline & $9,230 * * *$ & $8.611 * *$ & $-1,799$ & -2.714 & 2,397 & 1.785 \\
\hline $\begin{array}{l}\text { Au moins } 5 \text { ans post-Bac } \\
\text { (postgraduates) }\end{array}$ & 1,185 & 1.508 & $-3,400$ & $-7.990 * *$ & $-1,013$ & 3.995 \\
\hline Log(revenus autres) & & & & & & \\
\hline & $-0,0896$ & -0.116 & $-0,320$ & -0.172 & $-0,142$ & -0.0690 \\
\hline Taux Dependance Economique & $-5,189$ & -2.033 & $-6,143^{*}$ & $-7.304 *$ & $-13,00^{* * *}$ & $-16.96 * *$ \\
\hline $\begin{array}{l}\text { Durée totale } \\
\text { de travail domestique }\end{array}$ & & & & & & \\
\hline $\begin{array}{l}\text { Horaire flexible ou décalé: } \\
\text { Femme }\end{array}$ & $0,0029 *$ & -0.00064 & $-0,0054 * * *$ & $-0.0069 * *$ & 0,00200 & -0.0007 \\
\hline Homme & & -2.601 & & -3.573 & & $-4.503^{*}$ \\
\hline $\begin{array}{l}\text { Durée hebdomadaire de travail } \\
\text { rémunéré } \\
\text { femme }\end{array}$ & & -3.516 & & -2.952 & & -0.380 \\
\hline Homme & & -0.158 & & -0.147 & & $-0.200 *$ \\
\hline & & 0.191 & & $0.230 * *$ & & $0.317 * *$ \\
\hline Constante & & & & & & \\
\hline & $70,73 * * *$ & $74.92 * * *$ & $73,61 * * *$ & $64.25 * * *$ & $87,47 * * *$ & $79.10 * *$ \\
\hline$\overline{\mathrm{R}^{2}}$ & 0,159 & 0,116 & 0,155 & 0,15 & 0,170 & 0,256 \\
\hline $\mathrm{N}$ & 400 & 283 & 412 & 341 & 418 & 298 \\
\hline
\end{tabular}


Restreindre la focale aux couples dont homme et femme sont actifs (modèles 2), permet d'explorer comment les temps professionnels interagissent avec le partage du temps domestique. La durée de l'activité professionnelle masculine augmente la charge domestique de leurs compagnes à Paris et à Tokyo. L'allègement de la charge domestique des femmes lorsque leur durée de travail rémunéré s'allonge n'est sensible que dans les couples japonais mais cet effet reste insuffisant pour contrebalancer l'influence de la durée de travail du conjoint. Enfin, l'existence d'un horaire flexible et décalé pour les femmes japonaises contribue aussi à réaménager leur charge domestique à la baisse. Là aussi, on retrouve une interdépendance plus marquée entre temps professionnel et temps domestique de chaque conjoint dans les régimes conservateurs d'Etat-providence confirmant l'hypothèse 3. Enfin, le volume total de travail domestique n'a d'incidence réelle que dans les couples parisiens en contribuant à rééquilibrer le partage.

$\mathrm{Au}$ total, la contrainte temporelle liée à l'activité professionnelle des conjoints intervient particulièrement dans les couples tokyoïtes et, à un plus faible degré, français, alors que la logique de partage de l'activité domestique parait plus étanche aux temps consacrés à l'emploi dans les couples américains. Les temps d'activité entre conjoints y semblent moins substituables, comme si chaque partenaire avait davantage de liberté de décision par rapport à l'organisation de son temps personnel et sa déclinaison en activités, professionnelle, domestique, parentale (le cas échéant) et autre.

\section{Proximité et distance entre ces métropoles}

Pour éclairer notre quatrième hypothèse, il convient d'examiner si la forme de partage varie davantage en fonction des caractéristiques des couples qui diffèrent entre métropoles ou de la manière spécifique dont celles-ci influencent le partage selon la métropole d'appartenance.

On peut donc, par exemple examiner quelle répartition moyenne on obtiendrait pour les couples américains ou japonais si on leurs appliquait «le modèle de partage » obtenu pour l'échantillon des couples parisiens.

Les valeurs de partage se resserrent alors entre les échantillons par rapport aux répartitions prédites par les modèles, l'écart maximal est d'environ 6,5 points pour les modèles (1) et de 8,5 points pour les modèles (2) (Tableau 3). Ainsi, de par leurs caractéristiques familiales, de travail, d'éducation et de ressources, les japonaises sous un régime français de partage connaîtraient une charge relative supérieure d'environ 4,8 points à celles des parisiennes tandis que l'avantage des new-yorkaises par rapport à ces dernières serait de seulement 1,6 points de pourcentage. On peut en conclure que les couples parisiens sont proches des couples 
new-yorkais au plan de leurs caractéristiques individuelles et familiales. Cette proximité apparaît encore plus évidente dans le cadre de couples où homme et femme sont actifs (Modèle 2).

Inversement, on peut appliquer à la population des couples parisiens les structures de coefficients obtenues respectivement pour les couples japonais et américains. Les parisiennes assumeraient alors $73,8 \%$ du temps dévolu aux tâches domestiques à Tokyo et $64 \%$ de ce temps à New York (vs. $72.2 \%$ à Paris) (modèles 1) et respectivement 68,7\% et 64,2\% pour les estimations du modèle (2) (vs. 70,3 \% à Paris). La répartition obtenue par les couples biactifs français serait alors moins inégalitaire à Tokyo (modèle 2), illustration supplémentaire du poids des temps de travail professionnel pour les arbitrages dans ce pays. Il apparait ainsi que les mesures de partage du temps domestique dans le couple rapprochent les métropoles française et japonaise, alors que la structure de partage apparaît clairement plus égalitaire à New York $^{31}$.

Les différents «modèles de partage» semblent davantage contribuer aux écarts entre métropoles dans les modèles (1), mais dès lors que l'on se concentre sur les couples biactifs (modèles 2), ceux-ci se resserrent et ce sont les caractéristiques nationales des couples qui justifient principalement les écarts dans les prises en charge féminines du temps domestique.

En somme, les couples vivant à Tokyo et à Paris convergent par leurs modalités de partage du travail domestique mais se démarquent par les caractéristiques des hommes et femmes en couple, conformément à notre quatrième hypothèse. A cet égard, on retrouve la proximité entre le Japon et la France, régimes conservateurs d'Etat-providence dans la typologie d'Esping-Andersen, et leur distance à un régime libéral comme celui des Etats-Unis qui laisse place à un «modèle de partage » moins inégalitaire. On retrouve aussi le modèle familial « hybride » français décrit par Jane Lewis : ce sont les caractéristiques des parisiennes - en particulier leur positionnement au regard du travail rémunéré - qui leur permettent d'atténuer l'empreinte de modalités de partage « familialistes ». Ces résultats suggèrent que l'orientation de la politique familiale en France, plutôt généreuse en matière de conciliation entre travail et famille, amènerait davantage les hommes à s'exonérer de leurs responsabilités domestiques comme le notait Windebank $(2001)^{32}$ comparativement à un régime libéral, comme les EtatsUnis, qui promeut une égalité d'accès à l'emploi mais laisse à la responsabilité individuelle et

\footnotetext{
${ }^{31}$ Il est important de noter que ces conclusions seraient inchangées si on utilisait les modèles de partage calculés pour les couples new-yorkais ou tokyoïtes et qu'on les appliquait aux autres et de la même manière, si on retenait alternativement les caractéristiques de ces couples au lieu de celles des parisiens.

${ }^{32}$ Windebank, Jane. (2001), "Dual-earner couples in Britain and France: gender divisions of domestic labour and parenting work in different welfare states." Work, Employment and Society, 15(2), p. 269-290.
} 
au marché le soin de gérer l'articulation de la vie privée avec celle du travail. Ce dernier contexte semble ainsi contribuer, plus qu'ailleurs, à responsabiliser hommes et femmes dans les mêmes termes vis-à-vis de la sphère familiale et domestique.

Tableau 3 : Parts estimées du temps domestique à la charge des femmes

\begin{tabular}{llccc}
\hline$\%$ & & New York & Paris & Tokyo \\
\hline \multirow{2}{*}{ Modèle (1) } & Répartition prédite & 64,3 & 72,2 & 82,4 \\
& $\begin{array}{l}\text { Modèle de partage pour les couples } \\
\text { parisiens }\end{array}$ & 70,6 & 72,2 & 77 \\
& Caractéristiques des couples parisiens & 64 & 72,2 & 73,8 \\
\hline Modèle (1) & $\begin{array}{l}\text { A. Couple biactif à plein temps (a) } \\
\text { B. Couple biactif, femme à temps partiel (b) }\end{array}$ & 71,2 & 66,6 & 69,5 \\
& $\begin{array}{l}\text { C. Couple dont la femme est inactive ou au } \\
\text { chômage (c ) }\end{array}$ & 64,4 & 75,4 & 83,3 \\
& Répartition prédite & 66,4 & 82,9 & 88,5 \\
\hline \multirow{2}{*}{ Modèle (2) } & $\begin{array}{l}\text { Modèle de partage pour les couples } \\
\text { parisiens }\end{array}$ & 65,9 & 70,3 & 81,2 \\
& Caractéristiques des couples parisiens & 69,6 & 70,3 & 78 \\
\hline Modèle (2) & A. Couple biactif à plein temps & 64,2 & 70,3 & 68,7 \\
& B. Couple biactif, femme à temps partiel & 69,6 & 66,8 & 61 \\
\hline Répartitions relatives calculées à partir des estimations du tableau 2 & & 72,8 & 78,8 \\
\hline
\end{tabular}

(a) : Profil A, Couple biactif à temps complet, dont la femme contribue un peu plus que son mari aux revenus du travail du couple (TDE=0,02), qui délègue à un tiers le ménage, avec un enfant, dont la femme a moins de 30 ans pour un âge moyen des conjoints de 33 ans, dont la femme possède un niveau d'études supérieur ou équivalent à Bac +5 ans et le conjoint, un diplôme de Bac+ 4 ans, et avec une durée hebdomadaire totale de travail domestique de $17 \mathrm{~h} 00$ - et pour le modèle (2) dont la femme travaille 52 heures et le mari 53 heures, s'agissant du temps cumulé passé hors du domicile pour le travail.

(b) : Profil B, Femme travaillant à temps partiel ou/et en emploi irrégulier moins de $30 \mathrm{~h}$ par semaine contribuant pour environ $28 \%$ au revenu du travail total du ménage ( $\mathrm{TDE}=-0,45)$, dont aucune activité domestique n'est déléguée, dont la femme est âgée de 30 à 40 ans pour un âge moyen du couple de 38 ans, avec un enfant, dont la femme a un niveau d'études secondaires ou en-deçà et dont le conjoint a un diplôme de Bac+ 4 ans, et dont la durée totale hebdomadaire de travail domestique est de $22 \mathrm{~h} 00$ - - et pour le modèle (2) dont la femme travaille 25 heures et le mari 55 heures.

(c) : Profil C, Femme inactive ou au chômage qui contribue marginalement aux ressources du ménage (TDE=-0,75), sans délégation de travail domestique, avec 1 enfant, avec un âge moyen du couple de 43 ans et une épouse ayant elle-même plus de 40 ans, enfin avec une durée hebdomadaire totale de travail domestique de $26 \mathrm{~h}$.

\section{Quel partage de l'activité domestique selon le pays et le profil de couple?}

Une manière complémentaire d'apprécier les proximités entre pays consiste à s'intéresser aux répartitions relatives pour trois profils spécifiques de couples : ceux dont les conjoints sont tous deux actifs à temps plein, ceux dont la femme travaille à temps partiel et enfin les couples dont le mari est seul pourvoyeur de ressources (détails des profils en note du Tableau 3). 
A profil constant, les répartitions relatives du travail domestique au sein des couples varient sensiblement d'un pays à l'autre.

Pour le profil A où l'investissement professionnel féminin est fort, et si l'on suit les résultats du modèle 2 où sont comptabilisés les effets du temps de travail professionnel, c'est à Tokyo que l'on observe le meilleur équilibre dans la répartition entre les conjoints, confirmant à la fois le poids des ressources de l'épouse (monétaires et éducatives) dans la négociation du partage et l'impact de sa disponibilité temporelle. Parmi ces jeunes couples biactifs fortement investis dans le travail rémunéré, ce sont les new-yorkaises qui se retrouvent paradoxalement les plus mal loties avec 68 ou $71 \%$ du temps domestique à leur charge. Pour le profil $\mathrm{B}$ où les femmes ont un emploi à temps partiel, les ménages new-yorkais présentent cette fois la répartition la moins déséquilibrée (notamment dans le modèle 1 où $64 \%$ de l'activité domestique échoit à l'épouse) alors que l'inégalité de la répartition s'accroît fortement à Paris et à Tokyo relativement aux mesures du profil A. Cette forte accentuation au Japon rapproche l'investissement relatif de ces femmes de celui de leurs consœurs inactives (Profil C). Entre les profils B et C, c'est à Paris que le partage se dégrade le plus, les hommes n'assumant plus que $17 \%$ de l'activité domestique lorsque leur compagne est sans emploi contre près de $34 \%$ à New York.

Ces résultats confortent les précédentes observations : si la répartition du temps domestique est peu sensible aux investissements professionnels des conjoints dans la métropole américaine, à Tokyo et Paris elle varie respectivement de 19 et 16 points entre le premier et le dernier profil de couple (Modèle 1).

\section{Conclusion}

Dans les couples interrogés, il apparaît que c'est dans les couples new-yorkais que l'on trouve le partage le plus équitable devant les parisiens puis les tokyoïtes.

Le modèle conservateur d'Etat-providence, selon la typologie d'Esping-Andersen, imprime ainsi sa marque dans la division du travail domestique à Paris et à Tokyo. En effet, même si le partage des activités domestiques reste plus inégal dans les couples japonais que dans les couples français, les deux sociétés semblent se rapprocher sur les "modèles de partage ». C'est ce qu'on a pu établir en différenciant, dans nos analyses, certes limitées aux métropoles représentatives de chaque société, le poids des caractéristiques individuelles et celui des « modèles de partage » qui reflètent à la fois l'effet du cadre institutionnel et celui des référents culturels. En revanche, les couples parisiens et new-yorkais convergent au regard de 
leurs caractéristiques sociodémographiques, et de la figure dominante du ménage dont les deux conjoints travaillent à plein temps.

Le modèle familial de "monsieur gagne-pain ", dans la typologie de Lewis, apparait très présent dans les couples japonais tandis que le caractère «hybride » de celui-ci est confirmé pour les français. En effet, il se manifeste par la discordance entre les principes d'égalité qui marquent le monde du travail et les référentiels familialistes qui inspirent les politiques sociales et conduisent à soutenir et protéger les mères travailleuses, laissant la voie ouverte à la préservation des rôles de sexe et à une division traditionnelle des tâches domestiques (Crompton \& Lyonette, 2006) ${ }^{33}$. On ne retrouve pas en revanche dans les couples américains la force du modèle de «monsieur gagne-pain » que Lewis décrit pour le Royaume-Uni.

De fait, on a affaire à deux régimes libéraux où les modèles familiaux se sont construits dans des contextes socio historiques bien différents, avec en particulier au R-U le principe du salaire familial qui promeut un modèle à « un apporteur » resté dominant jusqu'au milieu du

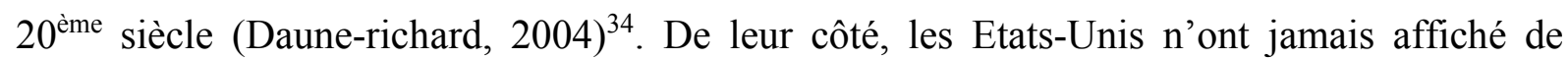
référent familialiste au côté des principes individualistes fondateurs. En revanche, ils ont développé des politiques de soutien aux mères en difficulté jusque dans les années 1970 qui voient l'avènement du workfare et le tournant de «l'adieu au maternalisme » (Orloff, 2006) ${ }^{35}$. Ce développement de «l'emploi pour tous» ne s'est pas accompagné d'un mouvement équivalent des aides à la parentalité et pourtant, au début des années 2000, les mères américaines étaient plus souvent en emploi que les françaises et interrompaient moins leur activité suite à une naissance (Périvier, 2009) ${ }^{36}$. La faiblesse des dispositifs publics et d'entreprises en faveur de la prise en charge des enfants d'âge préscolaire oblige le plus souvent les parents à recourir à des dispositifs privés ou à prendre eux-mêmes en charge leurs enfants. La plus grande proximité dans les investissements domestiques des conjoints que nous observons dans les couples new-yorkais peut ainsi se comprendre à l'aune de la faiblesse des dispositifs pour aider à la conciliation entre famille et emploi de la part tant des pouvoirs publics que des entreprises. En quelque sorte, nécessité ferait loi aux Etats-Unis, l'engagement fort des politiques publiques en faveur du droit des femmes au travail leur

\footnotetext{
${ }^{33}$ Crompton Rosemary, Lyonette Clare, (2006), "Work-life 'balance' in Europe”, Acta Sociologica, 49(4), p. 379-393.

${ }^{34}$ Daune-Richard Anne-Marie, (2004),'Les femmes et la société salariale : France, Royaume-Uni, Suède”, Travail et Emploi, 100, p. 63-77.

${ }^{35}$ Orloff Ann Shola, (2006), "L'adieu au maternalisme? Politiques de l'Etat et emploi des mères en Suède et aux Etats-Unis", Recherches et Prévisions, 83, p. 9-28.

${ }^{36}$ Périvier Hélène, (2009), "Les femmes sur le marché du travail aux Etats Unis. Evolutions et mises en perspective avec celles de la France et de la Suède", Revue de l'OFCE, 108, p. 49-84.
} 
ouvrant des opportunités d'emploi et de carrière qui ne peuvent être saisies que si les conjoints prêtent main forte à leur épouse dans la gestion des activités domestiques. Soulignons en outre l'importance du revenu de la femme compte tenu de la faiblesse de la protection sociale et de ressources de substitution : allocations chômage, aide au logement, aides sociales, santé etc.

On rejoint ici une limite de notre étude qui, portant sur ces couples des classes moyennes et supérieures, ne prend pas en compte les couches populaires et les familles à bas revenus. Or la réduction des inégalités de sexe qui caractérise les dernières décennies s'est faite dans un contexte d'accroissement des inégalités sociales et en particulier entre femmes qualifiées et non qualifiées. De ce point de vue, le régime républicain français peut s'avérer plus protecteur que le régime libéral ou le régime conservateur japonais.

Enfin au regard des composantes stratégiques de l'arbitrage autour de la répartition du travail domestique au niveau de la cellule familiale, à savoir les contributions individuelles au revenu du ménage et les temps de travail professionnel de chacun, on montre que ces dimensions, pour les individus étudiés appartenant aux classes moyennes et supérieures, jouent le plus fortement pour les couples tokyoïtes, à un degré moindre dans les couples parisiens et non significativement pour les couples new-yorkais. Leur incidence est ainsi amplifiée dans les régimes conservateurs et dans un pays comme le Japon où la division des rôles sociaux entre hommes et femmes reste encore culturellement marquée. 